\title{
L'éco-construction : quel positionnement pour les artisans ? 21
}

\author{
par Florence de Ferran ${ }^{22}$, Jeanne Lallement ${ }^{23}$ \\ et Isabelle Sueur ${ }^{24}$
}

Résumé

Quel positionnement pour l'artisan qui s'inscrit dans une démarche de développement durable ? A partir de cette interrogation, cette recherche explore les perceptions de l'éco-construction par les consommateurs. Après avoir présenté le cadre conceptuel, nous présentons la démarche exploratoire utilisée. 14 entretiens semi-directifs ont été conduits auprès de clients d'éco-artisans. Une analyse de contenu nous permet d'identifier ce qui caractérise un éco-artisan et les axes de communication utiles à leur positionnement.

Abstract

Which positioning for eco-craftsmen? We aim to identify customers' perceptions and the decision-making process in the area of eco-building. We first present the theoretical foundations. Then, to answer our research question we have conducted an exploratory qualitative research. 14 semidirective interviews of eco-craftsmen's customers were done which allowed us to identify eco-craftsmen attributes and the communication priorities to establish their positioning.

Le développement durable est à l'origine de la création et de la croissance de nombreux marchés à mesure que se développent les contraintes réglementaires et les incitations financières, et que s'affirment de nouvelles tendances de consommation. Parmi celles-ci, l'éco-construction renvoie à une relation harmonieuse des bâtiments avec leur environnement immédiat, un choix intégré des procédés et produits de construction, et un chantier à faibles nuisances. Comment les artisans pratiquant l'éco-construction peuvent-ils se différencier des artisans traditionnels ? Ce questionnement correspond à une problématique soulevée par un groupe de travail d'éco-artisans au sein du réseau "ArtisanatUniversité " mis en place à l'initiative de l'Institut Supérieur des Métiers. Précisément, les éco-artisans sont face à un dilemme : doivent-ils communiquer sur leur démarche écologique ? Ou doivent-ils plutôt se faire reconnaître avant

\footnotetext{
21. Une version antérieure des cet article intitulée «Quel positionnement pour l'artisan qui s'inscrit dans une démarche de développement durable ? Une étude basée sur les perceptions des particuliers dans le secteur de l'éco-construction ", a fait l'objet d'une communication aux 15èmes journées de Recherche en Marketing de Bourgogne, les 18-19 novembre 2010

22. Florence de FerRan, Maître de Conférences, Université La Rochelle, CEREGE-LR-MOS (EA 1722), florence.de_ferran@univ-Ir.fr

23. Jeanne Lallement, Maître de Conférences, Université La Rochelle, CEREGE-LR-MOS (EA 1722), jeanne.lallement@univ-Ir.fr

24. IsABELLE SueUR, Maître de Conférences, Université La Rochelle, CEREGE-LR-MOS (EA 1722), isabelle.sueur@univ-Ir.fr
} 
tout comme des professionnels du bâtiment ? Dans le premier cas, il s'agit d'afficher clairement leur capacité de réponse à un besoin de réduction de la pollution. Dans le second, il s'agit, après avoir réussi à se faire reconnaître comme professionnel de la construction, de proposer entre autres choses des solutions écologiques. La question de la mise en avant du savoir-faire du corps de métier, de la technologie et des matériaux utilisés qui sont spécifiques dans le cadre d'une démarche d'éco-construction se pose donc clairement.

La problématique soulevée est donc liée au positionnement des offres à dimension environnementale. Les recherches portant sur les attentes des consommateurs en la matière attestent de l'importance d'un positionnement centré sur le cœur de métier, la dimension écologique n'étant qu'une valeur ajoutée supplémentaire (Crane, 2001). On peut alors se demander si le marché de l'éco-construction répond à cette logique et si les pressions externes, qu'elles soient incitatives ou répressives, conduisent à une prévalence ou à une même importance de la dimension écologique sur le cœur de métier. Par ailleurs, la prise en compte de l'entreprise artisanale en tant qu'objet spécifique d'observation fait défaut (Picard, 2006 ; Paturel et Richomme-Huet, 2007).

Pour répondre à ces questionnements, il est important de faire un point sur les recherches qui ont défini l'artisan, l'éco-entrepreneur et les déterminants qui conduisent à le choisir (Pacitto et Julien, 2004 ; Allard et al., 2007 ; Paturel et Richomme-Huet, 2007). Puis, nous reviendrons sur le processus de choix mis en œuvre par le consommateur dans une situation qui demande un arbitrage entre son intérêt personnel et celui de la société, à savoir une prise de décision éthique. Ce cadre d'analyse permettra de nous interroger sur la pertinence d'un positionnement écologique pour conduire le consommateur à mettre en œuvre un projet d'éco-construction. Parallèlement, les connaissances relatives au domaine de la consommation de produits/services verts devraient nous renseigner sur les perceptions et motivations du consommateur.

Par la suite, la mise en œuvre d'une étude exploratoire nous permettra de déterminer le positionnement que les éco-artisans doivent adopter afin de rendre leurs offres attractives.

\section{Le réseau « Artisanat-Université @»}

Ce réseau est né en 2002, sur une initiative de l'Institut Supérieur des Métiers (ISM) et de différents partenaires, dont le Ministère de l'Economie et la Chambre des Métiers et de l'Artisanat. L'objectif affirmé du réseau est de combler le déficit de connaissances sur l'entreprise artisanale. Concrètement, dans chacune des régions où le dispositif est mis en place, des rencontres régulières ont lieu entre les artisans, la Chambre de Métiers et les universitaires. L'objectif est double : pour les artisans, il s'agit de prendre du recul sur leur activité, leur vision stratégique et de réfléchir à des solutions applicables à l'ensemble des entreprises artisanales ; pour les chercheurs, l'accès au terrain offert leur donne l'occasion de valider des axes de recherche. 


\section{L'éco-construction : quel positionnement pour les artisans?}

Le réseau "Artisanat-Université $®$ " Poitou-Charentes s'est mobilisé fin 2009 autour d'une problématique partagée par les artisans de la région et les chercheurs de l'Université de la Rochelle : l'innovation par la croissance verte et plus particulièrement l'éco-construction. Une douzaine d'artisans volontaires se sont inscrits dans ce processus de réflexion. II s'agit de dirigeants d'entreprises artisanales dans l'électricité, la plomberie, la menuiserie ou le chauffage, tous impliqués dans une démarche d'écoconstruction. Certains de ces artisans sont certifiés Eco-artisan par le CAPEB (Confédération de l'Artisanat et des Petites Entreprises du Bâtiment). Tous n'ont pas les mêmes pratiques, ni les mêmes convictions, mais ils partagent les mêmes interrogations ce qui permet une réflexion commune.

\section{Fondements conceptuels du processus de choix de l'éco- artisan}

\section{1. (Eco-) Artisan et processus de choix}

L'artisan a toujours évolué dans un système socio-économique de métiers oscillant entre une reconnaissance par un métier ou par un statut social (Louart, 1980, dans Paturel et Richomme-Huet, 2007). II a autant pu faire partie de corporations ou de communautés qu'être indépendant. Toutefois, le statut de l'entreprise artisanale se réfère à des éléments implicites tels que l'aspect manuel du travail effectué, la participation du propriétaire dirigeant au cycle productif, la qualification du chef d'entreprise dans le métier et la prise en compte d'une certaine tradition. C'est l'ensemble de ces éléments qui déterminent l'appartenance d'une entreprise au domaine artisanal (Paturel et Richomme-Huet, 2007). L'artisan est focalisé sur l'aspect technique de son métier, plus que sur l'écoute du marché. Ceci peut conduire à des difficultés lorsqu'il doit relever des défis de croissance (Pacitto et Julien, 2004) liés à une évolution de la demande comme cela peut être le cas pour les produits et techniques liés au développement durable.

La problématique de l'engagement des entreprises artisanales dans l'écologie et l'intégration de cette dimension à leur offre est plus ou moins saillante selon les caractéristiques de l'entreprise. Dans cette perspective, on peut s'appuyer sur différentes typologies. Celle proposée par Taylor et Walley (2004) repose sur l'orientation personnelle de l'entrepreneur et sur l'intensité des influences structurelles externes. Elle distingue «l'opportuniste innovateur » positionné sur une niche de marché et fortement influencé par les pressions externes notamment de nature réglementaire, du " champion visionnaire » doté d'une vision du futur durable dans une perspective de changements structurels de son métier, de « l'anticonformiste éthique » motivé par des convictions personnelles profondes, et de " l'éco-entrepreneur par accident » avec des motivations plus financières et moins personnelles. Les 12 artisans engagés dans notre groupe de travail se 
retrouvent dans un des quatre types. Cette dimension a donc été indirectement intégrée dans le cadre de notre recherche.

Par ailleurs, les artisans sont considérés comme un corps de métier qui s'adapte aux conditions locales en proposant des produits liés à l'identité territoriale de la région (Suire, 2004). En ce sens, il relève d'un développement durable car le fait d'avoir recours à des produits locaux conduit à limiter les impacts environnementaux de l'activité productive. Qu'en est-il du secteur du bâtiment? A priori, les matériaux employés par la plupart des artisans du bâtiment ne peuvent être qu'en partie des produits locaux.

Pour aller au-delà de cet ancrage local qui témoigne, parfois malgré l'artisan, d'une démarche environnementale, nous devons nous intéresser à l'éco-artisan. Il s'agit d'un artisan s'engageant à respecter un référentiel relatif à l'amélioration des performances énergétiques des logements (définition de la CAPEB). Sa perception par le consommateur devrait être commune, pour une part, avec celle de l'artisan à laquelle s'ajoutent des dimensions propres à l'écologie. Dans ce cadre, l'écologie peut être envisagée selon deux paradigmes distincts (Kilbourne, 1995) :

- Une vision anthropocentrique de l'environnement selon laquelle l'homme se perçoit dans un rapport de domination à la nature, celle-ci n'étant qu'un instrument de son bien-être. L'écologie et le développement durable de manière plus large ne s'envisagent que dans le cadre d'une évolution des technologies qui permettront à l'homme de continuer à satisfaire ses besoins en maîtrisant ses impacts environnementaux, voire en réparant ses impacts. Cette vision tournée vers le futur s'oppose à la seconde.

- Une vision écocentrique où l'homme ne se conçoit que comme un élément de l'environnement naturel. L'écocentrisme induit un retour à la frugalité car ce n'est qu'en limitant notre consommation que nous pourrons prétendre à un développement durable. Elle est donc ancrée dans la tradition et est davantage tournée vers le passé. II est alors possible que l'on retrouve cette dualité temporelle dans les discours des consommateurs ou au contraire que l'on identifie un discours très marqué dans l'une ou l'autre des orientations. Les associations à l'environnement et à l'éco-construction nous permettront de le déterminer.

De plus, on peut s'interroger sur l'importance des perceptions de nature environnementale qui seront associées à l'éco-artisan. Dans ce cadre, on peut faire un parallèle entre un produit respectueux de l'environnement et un écoartisan dans le sens où ce type de produits est considéré comme un produit « augmenté » c'est-à-dire un produit qui offrirait des bénéfices ou des services en plus, de manière à inciter à l'achat (Smith, 1990, dans Crane, 2001). Par analogie, on peut se demander si l'éco-artisan n'est pas un artisan « augmenté » 


\section{L'éco-construction : quel positionnement pour les artisans?}

de la dimension " éco », dimension qui lui apporte une valeur ajoutée sans être l'élément décisif du choix du consommateur.

L'ensemble de ces éléments nous amène à nous interroger sur l'importance de la dimension environnementale dans le positionnement de l'éco-artisan. Elle semble a priori secondaire, bien qu'intrinsèquement l'artisan soit proche des démarches de développement durable du fait de ses pratiques qui peuvent être perçues comme « traditionnelles ». II convient alors de s'interroger sur les déterminants du choix du consommateur pour une offre à caractère environnemental. II s'agit selon Allard et al. (2007) : du produit/service proposé, du savoir-faire, de la proximité relationnelle et de la proximité géographique qui ont déjà été mis à jour dans d'autres recherches (Siméoni, 1999 ; Julien, 2003 ; Fourcade et al., 2006, in Allard et al., 2007). Quant aux freins, ils sont majoritairement d'ordre relationnel. Une recherche conduite dans le domaine du BTP (Comets, 2006) ajoute que ce marché est associé à un fort risque perçu pour le consommateur car il s'agit de prestations de service à faible fréquence d'achat où l'expertise est rare. Ce constat amène les acheteurs à effectuer une recherche d'informations importante via le bouche à oreille et les sources d'informations secondaires, relativisant le poids des cautions extérieures (Comets, 2006). Enfin, au-delà de ces différents déterminants, nous nous intéressons à un modèle explicatif particulier : la prise de décision éthique.

\subsection{Prise de décision éthique}

Le recours à l'éco-construction par le consommateur peut être perçu comme une prise de décision éthique, son choix étant lié à un arbitrage entre son intérêt personnel et celui de la société à des degrés divers. Le modèle de Jones (1991), présenté en Figure 1, introduit les caractéristiques de la situation comme un déterminant principal de l'action. II les regroupe sous le concept d'intensité éthique qui désigne " l'impératif moral d'une situation », autrement dit sa gravité éthique. L'intensité éthique, qui est l'apport de Jones dans le modèle de prise de décision éthique, est composée de 6 éléments : l'ampleur des conséquences de l'action, le consensus social, la probabilité des effets, la proximité temporelle des effets, la proximité avec la cause et la concentration des effets. 


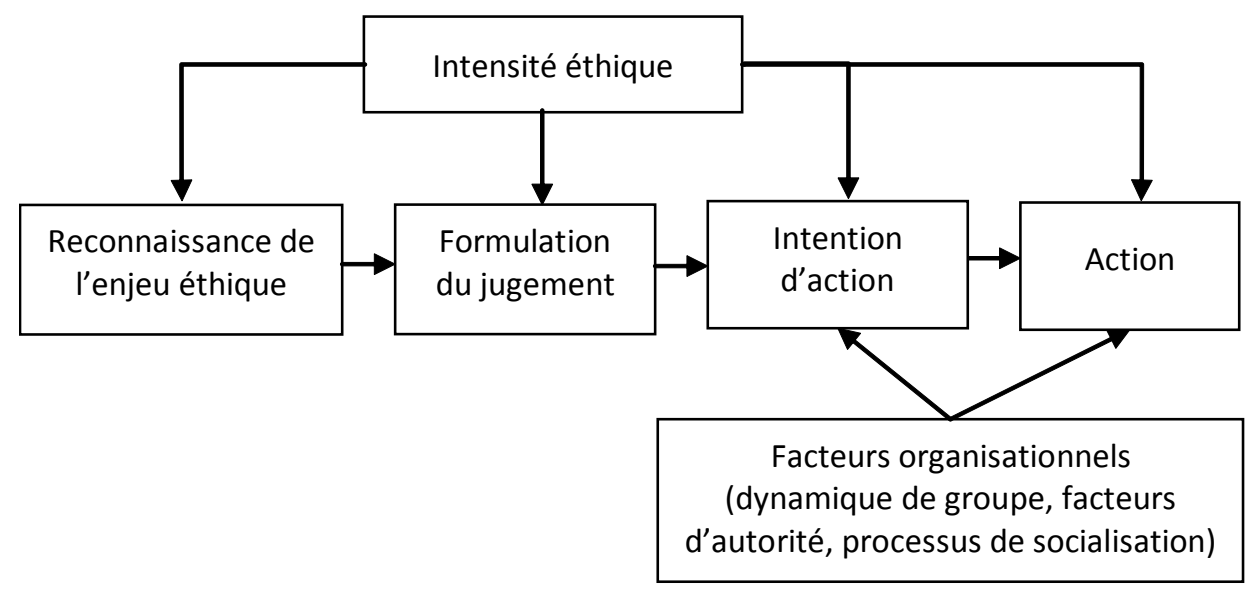

Figure 1 : La prise de décision éthique d'après Jones (1991)

Dans le contexte auquel nous nous intéressons, l'intensité éthique semble avoir un sens. Si nous reprenons ses 6 dimensions, nous constatons :

- Que l'ampleur des conséquences est une dimension qui peut être considérée dans le cas de la construction par l'utilisation d'un matériel qui pourrait avoir des conséquences importantes sur l'environnement au sens large ;

- Qu'il est probable qu'un individu choisissant l'éco-construction soit influencé par un consensus social car il fautun minimum de connaissances quant à l'existence de cette activité pour y avoir recours ;

- Que la probabilité des effets soit effective du fait de la toxicité des matériaux utilisés qui entraîne un risque élevé pour la santé ;

- Que la proximité temporelle des effets est envisageable car la mise en œuvre de travaux peut détériorer immédiatement l'environnement de la construction ;

- Que la proximité avec la cause c'est-à-dire la proximité de l'individu avec les effets est évidente puisque l'individu va vivre dans son habitation et sera donc en contact avec des matériaux qui peuvent être toxiques ; - Que la concentration des effets peut aussi être un enjeu car, dans certains cas, les pollutions liées à des travaux de construction peuvent toucher beaucoup de monde alors que, dans d'autres cas, elles peuvent ne concerner que quelques personnes.

Cette intensité éthique devrait être d'autant plus forte que $84 \%$ des individus se disent assez ou très sensibilisés à la qualité écologique de l'espace privé au sein duquel ils vivent, forment un ménage ou une famille, cet espace étant constitué du logement et du jardin, avec tous les êtres vivants qui les composent. Ce résultat est issu d'une étude de Sennes et al. (2007) portant sur les perceptions des composantes écologiques de l'écosphère familiale. 


\section{L'éco-construction : quel positionnement pour les artisans?}

Le modèle de prise de décision éthique de Jones (1991) a déjà été appliqué au comportement du consommateur et, plus particulièrement, à la consommation socialement responsable (François-Lecompte, 2006 ; Bernard, 2009). FrançoisLecompte (2006) en montre les limites et plus particulièrement la prévalence des facteurs situationnels dans la mise en œuvre de l'action. En effet, le comportement de l'individu est difficile à appréhender car il est fonction de la sensibilité de chacun pour la cause. II ne peut donc être prévisible qu'au regard des éléments situationnels. Toutefois, Bernard (2009) qui a appliqué ce modèle à l'étiquetage environnemental (considéré alors comme un élément situationnel) en montre la pertinence. Le caractère plus ou moins situationnel de l'objet étudié conduirait donc à une pertinence plus ou moins grande de ce modèle.

Dans le cadre de cette recherche, cette modélisation semble intéressante pour mieux comprendre les déterminants du comportement du consommateur qui auront un impact sur la présentation de l'offre des éco-artisans. Notre étude exploratoire aura pour objectif de les circonscrire. Au-delà de ces éléments, nous pouvons également nous interroger sur les ressorts du comportement à travers les motivations et freins à l'achat de produits/services respectueux de l'environnement.

\subsection{Déterminants à l'achat de produits/services respectueux de l'environnement}

Les produits verts encore appelés écoproduits, produits propres, produits pro environnement, produits écologiques ou produits respectueux de l'environnement, sont des produits dont la production, la distribution, la consommation et l'élimination, respectent l'environnement (Kovarski et Renault, 2000). On remarque que cette définition appelle à un polymorphisme des comportements d'achat de produits verts compte tenu de la profondeur du concept. Nous nous limiterons aux produits ayant un attribut « vert » tels que les produits sans agents polluants, les produits recyclables ou biodégradables, qui ont été les plus étudiés.

Une des premières tentatives d'identification du profil du consommateur de produits écologiques fut effectuée par analogie au consommateur socialement responsable par introduction du concept de responsabilité écologique (Kinnear, Taylor et Ahmed, 1974). Un consommateur est défini comme écologiquement responsable quand son attitude exprime un intérêt pour l'écologie et que son comportement d'achat est cohérent avec la conservation des écosystèmes. Pour Straughan et Roberts (1999), il s'agit d'un consommateur qui achète des produits qui ne dégradent pas l'environnement ou qui le dégradent moins que les autres produits, et/ou d'un consommateur qui tente de réduire son impact personnel sur l'environnement. L'idée sous-jacente à cette consommation est donc la préservation de l'environnement. L'individu qui fait appel à un artisan écoconstructeur correspond a priori à ce profil. 
Par ailleurs, la consommation écologiquement responsable va au-delà de la seule satisfaction des besoins et permet au consommateur d'exprimer ses valeurs (Kréziak et Valette-Florence, 1997). De nombreuses recherches ont montré que les valeurs universalistes sont des antécédents aux attitudes environnementales (Thøgersen et Ölander, 2003 ; Robinot et Giannelloni, 2009). De plus, différents buts conduisent les individus à acheter des produits verts : le plus manifeste est la volonté de préserver l'environnement par amour pour la nature et afin d'assurer de bonnes conditions de vie future (Kréziak, 1998). L'acheteur de produits verts cherche ainsi à exprimer sa responsabilité vis-à-vis de la collectivité, sa volonté de participer à un développement durable, son altruisme (Kréziak, 1998 ; Follows et Jobber, 2000). Cette consommation fait également état de motivations personnelles avec une recherche du bien-être, du plaisir (Kréziak, 1998) et une volonté de préserver sa santé (Suchard et Polonsky, 1991 ; Kréziak, 1998).

Les recherches portant sur le consommateur de produits/services verts montrent que les idées de responsabilité, d'altruisme, de développement durable, sont associées à cet achat, tout comme celles de bien-être, de plaisir et de préservation de sa santé. Par conséquent, cet acheteur serait conduit par des motivations de nature individuelle et sociale.

L'éco-construction reste un secteur qui requiert des dépenses importantes de la part du consommateur. Dans ce cadre, on peut considérer que l'engagement environnemental n'est qu'un plus à sa démarche. Comme le dit Giannelloni (1998): « dès lors que l'ensemble des coûts relatifs au choix surpassent le bénéfice psychologique lié au sentiment de protéger l'environnement, l'attribut vert (...) devient secondaire » (pp.64). Nous pourrons vérifier dans le cadre de notre étude exploratoire si effectivement la dimension environnementale est secondaire par rapport à d'autres dimensions comme le savoir-faire, par exemple.

\section{Méthodologie}

Notre recherche s'appuie sur une méthodologie qualitative qui permet d'appréhender le pourquoi et le comment des évènements dans des situations concrètes (Wacheux, 1996). Ce choix s'explique par la quasi-inexistence de connaissances théoriques liées à l'émergence du phénomène étudié.

La collecte des données a été réalisée par le biais d'entretiens semi-directifs. Un guide d'entretien a fourni aux trois chercheurs un cadre de déroulement commun aux différents entretiens. Il est réalisé sur la base des interrogations managériales et de la problématique de recherche posée. Celui-ci est composé de 4 thèmes abordant la définition de ce qu'est un artisan, un éco-artisan et l'éco construction, ainsi que le récit du processus de choix des répondants, de l'identification de leur besoin jusqu'à l'achat. Le guide d'entretien volontairement peu détaillé a permis 


\section{L'éco-construction : quel positionnement pour les artisans?}

d'offrir à l'enquêteur une certaine souplesse quant à l'ordre des questions et la possibilité de relances sur le rôle des labels, les sources d'informations utilisées ou encore sur les prescripteurs spontanément énoncés.

Le terrain s'est naturellement porté vers les clients des 12 éco-artisans présents dans le groupe. Chaque corps de métier a proposé une liste de clients récents. Le choix a été fait de réaliser les entretiens au domicile de 10 d'entre eux. Dans une volonté de comparaison, nous avons également interrogés 4 clients qui ont eu recours à des artisans classiques. Chaque entretien a duré entre trente minutes et une heure trente. Le petit nombre d'individus interrogés permet de procéder à une méthode d'analyse manuelle des entretiens, longue et fastidieuse, tout en satisfaisant aux critères de saturation. Elle s'est faite par le biais d'une analyse de contenu thématique. Les 14 entretiens de consommateurs enregistrés et retranscrits exhaustivement donnent un ensemble de 40315 mots. L'analyse du corpus vise trois objectifs : la clarification du concept d'éco-artisan, la catégorisation des variables et des thèmes centraux, et la modélisation des liens de causalité. Après une première organisation des données sous forme de fiche de synthèse permettant une récapitulation globale de chaque interview (Miles et Huberman, 2003), le processus général d'analyse se rapproche de la méthode de Spiggle (1994) qui propose une classification et une description de l'analyse par sept opérations élémentaires.

\section{Résultats}

L'analyse du corpus obtenu permet de tirer des conclusions quant aux éléments suivants : 1 / le rapport des individus à l'environnement, 2 / leurs perceptions des artisans, éco-artisans et de l'éco-construction, $3 /$ le processus de choix mis en œuvre pour recourir aux services d'un éco-artisan.

\subsection{Rapport de l'individu à l'environnement}

La nécessité de respecter, de préserver l'environnement est clairement présente au sein du discours des interviewés par le souhait de minimiser ses impacts sur l'environnement, de limiter sa pollution. Dans ce cadre, les évolutions technologiques ne sont pas remises en cause. Pour autant les techniques de construction anciennes, alliant le respect de l'environnement et le maintien du confort suscitent l'intérêt. L'individu se situe alors davantage dans une vision anthropocentrique de son environnement. Ceci est d'autant plus manifeste que ses motivations à avoir recours à l'éco-construction - et donc à des techniques respectueuses de l'environnement - sont orientées vers lui avec le souhait de vivre mieux, de vivre dans un environnement sain (« Nous préserver nous - parce que c'est la partie centrale - et l'environnement »). 
On peut affirmer qu'aucun répondant n'a présenté un rapport écocentrique à l'environnement ; ils n'ont jamais considéré que le respect de la Nature était important dans le sens où une modification de cet environnement pouvait impacter leur vie. Ils ont donc toujours eu une vision de domination, de supériorité vis-à-vis de l'environnement.

Le rapport à l'environnement

«On n'est pas des ayatollahs de l'écologie. On va faire du sain. Voilà, pour faire simple on va faire du sain ». "Faire les choses au mieux et à minima par rapport aux impacts sur l'environnement (...). Faire des choix techniques et dans la construction qui garantiront à terme un bilan énergétique qui soit inférieur au bilan énergétique d'une maison classique ». "Qui génère le moins de consommation d'énergie possible en s'intégrant bien dans son environnement et en ayant un super confort pour les utilisateurs ». "On ne peut pas dire que l'écologie dans ce domaine-là ce soit un retour à la bougie parce que c'est quand même hyper technique. C'est un vrai petit ordinateur » (à propos d'une chaudière à granulés bois).

\subsection{Artisans, éco-artisans et éco-construction}

Sans surprise, ce qui fait la qualité d'artisan est conforme aux éléments identifiés dans la littérature. Participent à la définition d'un artisan 1/ l'aspect manuel et technique du travail effectué, 2/ le fait que l'artisan soit également le chef de l'entreprise (de très petite taille) et $3 /$ que l'activité possède un ancrage local notamment au regard de l'origine des matériaux utilisés. Au-delà de ces éléments, on identifie également 4/ l'importance de la dimension relationnelle ainsi qu'un certain parallèle avec le travail que pourrait réaliser un artiste. Nous sommes donc face à une perception réellement qualitative de l'artisan et de son travail.

\section{De l'artisan...}

« Une personne qui a un savoir, un savoir-faire, qui a normalement la maîtrise de l'achat des matériaux, de la préparation de son chantier, de la réalisation, du suivi permanent clientèle sur la progression du chantier, des réunions de suivi de chantier, et puis qui est soucieux de la qualité ». «Qui connaît son métier, qui intervient dans un rayon assez proche (...) surtout qu'il tienne compte de ce qu'il y a autour de lui en termes de matériaux ». "Plutôt une micro-entreprise ou une petite entreprise ». "Qui crée quelque chose (...). II produit une œuvre ». " C'est quelqu'un qui est proche du client, où il n'y a pas d'intermédiaire ».

\section{...à l'éco-artisan}

« C'est quelqu'un qui choisit ses matériaux par des valeurs écologiques (...) une éthique différente de n'importe quel artisan ». « Un artisan tel que je l'ai défini auparavant, avec en plus une dimension orientée vers l'écologie et/ou le développement durable ». "En utilisant les matériaux proches, comme autrefois en fait, et en utilisant des produits non nocifs, ni pour les gens ni pour les bêtes ni pour tout ce que l'on veut, de façon à consommer le moins d'énergie possible ». " (II utilise) ... des matériaux de base naturelle... que l'on peut trouver directement dans la nature, soit par le biais d'une petite transformation mécanique ou un peu semi-industrielle, sous forme de produits manufacturés ». "Ce n'est pas qui respectait l'environnement, mais en tout cas qui permettait de moins dépenser d'énergie ». « II intervient dans un rayon assez proche ». "Ce n'est pas un esclavagiste $»$. 


\section{L'éco-construction : quel positionnement pour les artisans?}

Spontanément la dimension écologie/respect de l'environnement est associée au terme " éco-artisan », que les répondants aient connaissance ou non de ce terme. Ceci est d'autant plus intéressant que l'amélioration des performances énergétiques des logements à laquelle renvoie officiellement le terme éco-artisan peut tout à fait techniquement reposer sur la mise en œuvre de techniques/ matériaux non respectueux de l'environnement. Un interviewé a d'ailleurs soulevé le paradoxe en soulignant les difficultés de recyclage des panneaux solaires. D'autres vont même jusqu'à identifier des produits/matériaux qui, selon eux, ne sont pas respectueux de l'environnement même si les pouvoirs publics les présentent comme tels ("Les pompes à chaleurs ou climatisations réversibles ne sont pas, en soi, des produits respectueux de l'environnement »).

Plus en détail, trois dimensions distinguent l'artisan de l'éco-artisan :

- Leur activité utilise des méthodes et des techniques orientées vers l'écologie ;

- Les matériaux utilisés, qui se trouvent dans l'environnement local proche, ont une valeur écologique (le bois par rapport au plastique par exemple), et permettent de dépenser moins d'énergie ;

- L'éco-artisan est supposé respecter un cahier des charges particulier, avoir une certaine éthique ou philosophie propre à son positionnement écologique.

II ressort donc des verbatim analysés que le fait de considérer les éco-artisans comme des « artisans plus » c'est-à-dire augmentés d'une dimension écologique, semble tout à fait pertinent. Toutefois, la capacité à mettre en œuvre certaines techniques spécifiques est bien un attribut déterminant dans le processus de choix de tel ou tel artisan. Dans ce cas, il n'y a pas de convergence avec le concept d'écoproduit pour lequel cette dimension supplémentaire incite à l'achat sans pour autant être déterminante. Les spécificités de l'échantillon interrogé et le caractère pionnier des démarches mises en œuvre par les interviewés peuvent contribuer à expliquer cette réalité.

L'analyse du sens donné au terme " éco-construction » par les sujets interrogés correspond à une réelle interrogation managériale des artisans du réseau Artisanat-Université, le préfixe " éco " pouvant être synonyme d'écologie comme d'économie. De façon unanime, les consommateurs y voient une double signification, réunissant l'écologie et l'économie. Le terme " éco-construction », beaucoup plus connu que l'éco-artisan, revêt des dimensions complémentaires autour de :

- L'écologie, avec le respect de l'environnement et l'utilisation de matériaux locaux et sains ;

- L'économie d'énergie et l'économie monétaire basée sur le calcul à long terme des matériaux économes en énergie ; 
- La « démarche globale » qui caractérise l'éco-construction. "C'est comme manger bio!» ;

- Une démarche qui s'inscrit dans le temps. On peut à ce propos noter la dimension temporelle paradoxale associée à l'éco-construction où passé et futur se rejoignent. Les énergies nouvelles sont aussi décrites comme des énergies anciennes, le savoir-faire associé rappelle les " techniques ancestrales " et les matériaux sont ceux " d'autrefois". Dans ce retour au passé, le futur est présent par la technologie.

\begin{tabular}{l} 
L'éco-construction \\
«....matériaux moins coûteux à produire, plus pérennes, plus résistants ». « Par exemple, une \\
maison en bois, une maison à la chaux ». " C'est aussi utiliser des chasses d'eau qui recyclent \\
en partie l'eau, utiliser un système de chauffage plus respectueux de l'environnement ». " Tout ce \\
qui est éco c'est de l'ancien que l'on a remis au goût du jour, des choses qui existaient il y a très \\
longtemps... C'est deux mondes qui se relient, on dirait un serpent qui se mord la queue ». "C'est \\
la mise à jour d'un savoir ancestral ». " On ne peut pas dire que l'écologie dans ce domaine-là ce \\
soit le retour à la bougie parce que c'est quand même hyper technique ». \\
\hline
\end{tabular}

Si l'on admet que les éco-artisans sont des artisans possédant les compétences nécessaires pour déployer des techniques d'éco-construction, alors l'écoartisanat est susceptible de renvoyer à une grande palette de métiers. En effet, lorsqu'on les interroge sur ce qu'est l'éco-construction, parmi les réponses, sont clairement identifiées les différents métiers du BTP, sous réserve que la dimension environnementale soit présente : un maçon, un charpentier, un plombier, un électricien, un menuisier ont donc la légitimité pour se prévaloir de cette (ces) compétence(s) spécifique(s).

\subsection{Motivations du recours à l'éco-construction}

Nous traiterons aussi bien des motivations sous-jacentes à la volonté de mettre en œuvre une démarche d'éco-construction que de celles qui expliquent le recours à un éco-artisan. Les deux sont en effet inextricablement liées, les éco-artisans possédant les compétences nécessaires à la mise en œuvre d'un projet d'écoconstruction ( II va comprendre comment la maison a été pensée, le système qui est déjà existant, et ensuite, il pourra, en fonction des possibilités, y amener des points intéressants en éco-construction »). En revanche, les clients n'ont pas explicitement recherché un éco-artisan ou un artisan qui fait de l'éco-construction mais davantage des artisans qui étaient à même d'utiliser telle ou telle technique, d'atteindre tel ou tel objectif, ou de les conseiller en matière d'éco-construction.

Les individus qui ont eu recours à des éco-artisans ont été conduits par différents types de motivations : des motivations fonctionnelles, hédonistes et d'autoexpression, au sens de Joannis (1965) et Roy (1994). Pour les premières, il s'agit par exemple, lors de travaux de rénovation, donc sur des chantiers partiels (versus une construction d'habitat de $A$ à $Z$ ou une rénovation très lourde), de mettre 


\section{L'éco-construction : quel positionnement pour les artisans?}

en œuvre un système de chauffage qui permette de générer des économies d'énergie et donc des économies au niveau de la facture énergétique du foyer. Certains, anticipant une évolution de la législation en faveur de l'utilisation de matériaux plus respectueux de l'environnement, estiment procéder de manière rationnelle en utilisant par exemple de la laine de chanvre plutôt que de la laine de verre en guise d'isolant : " C'est aussi une démarche économique, c'est-àdire que c'est de se dire : O.K. cela va coûter plus cher mais dans vingt ans, si je veux revendre [si on ne revend que dans vingt ans et non pas dans trois], j'aurai une maison qui n'aura pas de laine de verre et les normes auront évolué sur les isolants [cela va être interdit à la revente bientôt, pas bientôt mais cela va finir par arriver] ». Les facteurs situationnels liés à la conjoncture économique sont dès lors primordiaux ( « J'ai pris ma décision au moment où le pétrole était à son pic le plus élevé »).

Quant aux motivations hédonistes, elles répondent à une volonté de se faire plaisir en ayant recours à ce type de rénovation/construction. Dans ce cadre, l'individu mobilise des motivations et valeurs orientées vers lui ; il recherche, par exemple, " une maison qui soit agréable à vivre », " qui soit saine ». L'écoconstruction est alors envisagée dans le cadre d'une démarche personnelle, mais aussi, dans le cadre d'une démarche globale qui témoigne de son style de vie. En ce sens, le recours à un éco-artisan est aussi lié à des motivations d'autoexpression. II s'agit de bâtir un projet qui soit en harmonie avec soi : " envie de ruralité, de choses saines ", dans " un tout cohérent (avec...) des principes ", "du militantisme ». Ces motivations d'auto-expression peuvent être associées au domaine motivationnel de la spiritualité selon la typologie de Schwartz et Bilsky (1987) qui témoigne d'une recherche d'harmonie intérieure, d'un souhait de donner un sens à sa vie. II traduit une orientation sociale des motivations de l'individu, tout comme l'universalisme qui est également un ressort des individus ayant recours à l'éco-construction, notamment ceux qui souhaitent préserver l'environnement ou qui souhaitent vivre en harmonie avec la nature.

Le choix de recourir à l'éco-construction pourrait donc être envisagé comme un arbitrage entre des motivations de nature sociale et de nature individuelle, d'où l'intérêt de considérer le modèle de prise de décision éthique qui témoigne de cet arbitrage.

\subsection{Processus de choix}

Nous avons interrogé des individus une fois leur projet de construction/rénovation réalisé. Si l'on considère les six dimensions du concept d'intensité éthique introduit par Jones (1991) dans le modèle de prise de décision éthique, quatre d'entre elles ont été évoquées spontanément par les répondants comme des facteurs influençant leurs décisions d'achat. Ainsi : 
- L'ampleur des conséquences est sous-jacente au choix des individus. Dans des réponses souvent pudiques, ils reconnaissent que les solutions non écologiques, à base d'énergies non renouvelables, ne " vont pas dans le bon sens. II va falloir faire demi-tour », ce qui justifie leur choix d'une solution écologique.

- De la même façon, la probabilité des effets et la proximité avec la cause, notamment liées aux risques sur la santé de certains matériaux et aux bénéfices d'autres, sont des notions reprises dans tous les discours. "On ne respire pas tous les composants chimiques qui sont dans les produits que l'on avait jusqu'à dix ans ».

- En outre, la concentration des effets se décline par les multiples solutions écologiques envisagées, touchant tous les domaines de la maison, depuis les murs, les fenêtres, le bois utilisé, la circulation de l'air, la peinture ou encore les matériaux isolants.

Les deux autres dimensions que sont le consensus social et la proximité temporelle des effets sont évoquées de manière moins unanime :

- le consensus social n'a pas été évoqué dans les entretiens. En revanche, les répondants nous ont dit souffrir d'une image négative, due sans doute à leur décision " avant-gardiste » : "Le seul point négatif c'est qu'ici je suis prise pour une folle dans le village quand même (...), la poule, la dame au granulés (qu'elle utilise comme combustible pour sa chaudière)... mais bon, quelques-uns reviennent sur leurs idées premières ". La reconnaissance par le public de leur démarche est récente : " cela commence à ne plus être un truc folklorique, de vieil écolo baba-cool ! », et ce non consensus social pourrait être envisagé comme un facteur pénalisant la démarche écologique : " on a un peu l'impression de nager à contre-courant ».

- En outre, la proximité temporelle des effets n'est pas un élément qui a influencé directement le choix, ce qui est sans doute à mettre en lien avec la récence des technologies utilisées. Les sujets reconnaissent le manque de recul des artisans et de la profession, en général, sur les conséquences environnementales des matériaux utilisés. Deux exemples sont repris dans les discours : les panneaux photovoltaïques " là où j'ai des doutes, c'est sur tout ce qui est photovoltaïque » et la pompe à chaleur : « il s'avère que si l'on creuse un peu... est-ce vraiment si écologique ? dans l'éco-construction, on est sans arrêt rattrapé par le truc !».

Au-delà de ces déterminants du choix, d'autres caractéristiques du processus de choix ont pu être relevées dans les discours des répondants. Tout d'abord, le choix d'un éco-artisan est lié à un processus de recherche d'information long, parfois complexe et fastidieux. Si le bouche à oreille est la première source d'information utilisée (citée 13 fois sur 14 comme la source d'information prioritaire) 


\section{L'éco-construction : quel positionnement pour les artisans?}

pour trouver le bon artisan, les revues spécialisées (Habitat Naturel, Maison Ecologique), les annuaires spécialisés et Internet sont régulièrement cités. Tous les éco-consommateurs interrogés ont utilisé de multiples sources d'information complémentaires. On note dès lors des phases de recherche d'information, allant de quelques mois à deux années dans un cas. Ce processus de recherche est cohérent avec le fort risque perçu inhérent à cette situation d'achat. II constituera certainement un déterminant de l'intensité éthique en développant l'expertise de l'individu. Dans ce cadre, une partie des individus interrogés sont apparus comme des clients experts de l'éco-construction qui ont souvent eu plus de mal à trouver un artisan acceptant de travailler, par exemple, un nouveau matériau plutôt qu'à choisir entre plusieurs artisans. Cette particularité est sans doute due à notre échantillon obtenu directement auprès d'artisans déjà fortement impliqués dans la démarche écologique et disposant d'un portefeuille de clients qui leur ressemble.

De plus, la proximité avec le chantier constitue un déterminant important dans le choix de l'artisan. La dimension locale qui - comme nous l'avons vu dans la littérature - fonde l'identité de l'artisan, est cohérente avec les attentes des consommateurs qui cherchent avant tout une prestation de proximité. En outre, le prix n'est pas un critère de choix déterminant car les clients savent bien que l'éco-construction engendre un surcoût immédiat. Tout au plus intervient-il une fois la première sélection d'artisans effectuée. Enfin, un répondant dit avoir pris en compte le climat social des entreprises avec laquelle il a choisi de travailler.

Choisir un Eco-artisan

« Nadia m'a fait rencontrer Christophe parce que justement je lui avais parlé de ma démarche ». « J'avais trouvé une association qui s'appelait FUTUROBOIS qui (...) faisait une espèce de petite liste des artisans (...) c'était une association, donc je me suis dit : ce n'est pas une entreprise, il n'y a pas quelque chose de commercial derrière (...). Ça devait être par connaissance que j'ai eu cette adresse ». "II y a ceux qui ont répondu un peu vite (...) qui m'ont relancés* ». "A l'époque on avait un peu l'impression de ramer à contre-courant ». "C'est la proximité en fait ».

* Par opposition à des artisans, qui ayant été sollicités, n'ont jamais donné suite.

Même si les clients reconnaissent qu'il n'a pas été facile de mener à bien leur projet (difficultés à s'y retrouver dans les solutions techniques disponibles, difficultés à trouver en local un artisan compétent) un consensus très fort se dégage quant à la non-utilisation de labels ou autre référentiel pour trouver l'artisan adéquat. Trois cas de figure sont identifiés :

- Les labels ne sont pas connus du public (qui pourtant dans le cas présent est constitué de clients qui sont devenus experts);

- Certains labels sont connus, mais les clients ne savent pas véritablement ce qu'ils recouvrent ;

- Il y a une méfiance quant à la confiance que l'on peut accorder à ces labels (« une journée de stage payée $1000 €$ et puis on a le label »). 
Un véritable effort de pédagogie semble donc souhaitable, la dimension environnementale de l'artisan étant véritablement recherchée par les individus interrogés, mais difficilement identifiable par ces derniers. Ceci est d'autant plus important que les évolutions attendues de la réglementation en matière d'écoconstruction, ainsi que le poids croissant des valeurs liées au développement durable en France ${ }^{25}$ vont conduire de plus en plus de clients à se tourner vers ces techniques de construction.

\section{Apports, implications managériales, limites et voies de recherche}

Cette recherche se situe dans une perspective exploratoire, d'examen des perceptions des particuliers dans le secteur de l'éco-construction. Ses apports conceptuels résident dans l'exploration d'un comportement d'achat vert impliquant et dans la mise en perspective du modèle de décision éthique, pour des achats impliquant, d'un montant unitaire élevé, et avec des processus de décision extensifs.

Dans ce contexte, la distinction entre la vision anthropocentrique et écocentrique n'apparaît pas telle que définie dans la littérature, nos répondants ayant toujours fait état d'une perception de domination de la Nature. Par ailleurs, l'éco-construction traduit le désir de conciliation temporelle recherchée dans les démarches de développement durable entre le passé et le futur même si cela peut être paradoxal pour certains consommateurs. En revanche, leurs perceptions de la spécificité des artisans et la définition de l'éco-artisan comme un " artisanplus » semblent confirmées. De façon plus globale, le modèle de décision observé dans le choix de l'éco-construction présente les caractéristiques de la prise de décision éthique telle que définie par le modèle de Jones (1991).

Sur un plan managérial, cette recherche a de nombreuses implications qui se traduisent par des recommandations concrètes formulées auprès des artisans et des organismes institutionnels parties prenantes de ce réseau. Ainsi, la perception temporelle ambiguë associée à l'éco-construction incite à recommander aux artisans d'adopter un positionnement mixte, entre professionnel de la construction et de l'écologie. La vision parfois passéiste de l'écologie peut poser problème au niveau des perceptions des consommateurs qui pourraient associer cette offre à une offre artisanale d'autrefois et n'y verraient que la dimension écocentriste de retour à la frugalité et à un moindre confort. D'après une consommatrice interrogée qui a eu recours à des artisans non éco-constructeurs : «C'est un peu trop. J'aime bien ce qui est moderne, pratique donc je n'irai pas jusque-là (faire de l'éco-construction). Je tiens quand même à mon confort ! ». De même, comme

25. Les français et le développement durable, enquête TNS Media Intelligence réalisée en février 2008 pour Ethicity et Aegis Media Expert en partenariat avec l'ADEME. 


\section{L'éco-construction : quel positionnement pour les artisans?}

nous l'avons souligné, les personnes qui ont eu recours à l'éco-construction ont souvent une mauvaise image du fait d'une consommation encore marginale qui peut être encore perçue comme régressive (" c'est des écolos radicaux »). Les éco-artisans doivent donc être conscients de cette perception qui est associée à leur marché et doivent tenter de démocratiser leur offre en insistant sur des dimensions d'ordre hédoniste. En outre, d'autres recommandations spécifiques, liées à l'importance des sources d'information informelles ou au manque de clarté des labels, ont été développées.

Plus généralement, il semble que le travail initié au sein du groupe ArtisanatUniversité a atteint ses objectifs de co-construction de la connaissance. Sur le plan opérationnel, les artisans ont pris le temps de "poser leurs problèmes » et ont obtenus des éléments de réponse aux problèmes identifiés. Dans une perspective plus conceptuelle, cet observatoire des pratiques managériales a fourni une première étude d'un cas tout à fait intéressant.

Ce travail n'est pas exempt de limites. Notre recherche s'est appuyée sur le discours d'un groupe restreint de clients des artisans de notre groupe de réflexion. Une prochaine étape, auprès d'autres types de clients, permettra de travailler dans un cadre moins contextualisé.

Sur le plan méthodologique, cette recherche exploratoire ouvre plusieurs pistes afin d'explorer de façon plus systématique et moins personnalisée, les potentiels offerts par l'éco-construction. L'utilisation d'un cadre conceptuel plus large tel que la chaîne de valeur appliquée à une problématique de RSE (Hoffmann et Saulquin, 2009) permettrait de répondre à la problématique des éco-artisans de manière plus globale en tenant compte de l'ensemble des ressources et décisions sur lesquelles ils peuvent agir. En outre, il serait intéressant, au-delà de la réalisation d'entretiens semi-directifs, de compléter notre travail en nous appuyant sur des récits de vie afin d'approfondir notre compréhension des démarches des clients étudiés.

Les concepts de proximité (géographique ou interpersonnelle) et de relation au temps (concilier le passé et le futur) nécessitent de plus amples développements. De la même manière, une recherche empirique basée sur le modèle de la prise de décision éthique paraît appropriée à cet objet d'étude.

\section{Bibliographie}

ALLARD F., AMANS P., BRAVO K., LOUP S. et DESCARGUES R. (2007), « Quand les clients regardent l'entreprise artisanale : les leçons d'une étude empirique ", $5^{\text {ème }}$ Congrès de l'Académie de l'Entrepreneuriat, Sherbrooke, Canada.

BERNARD Y. (2009), " L'étiquetage écologique des produits en magasin ", Revue Française du Marketing, 221, 1/5, pp. 63-83.

COMETS C. (2006), « Comment trouver un professionnel de confiance pour faire des 
travaux chez soi ? Dispositifs personnels vs. Dispositifs impersonnels ", Actes du Congrès AFS, RT12 Sociologie économique, Bordeaux.

CRANE A. (2001), " Unpacking the ethical product », Journal of Business Ethics, Vol. 30, n4, pp. 361-373.

FOLLOWS S.B. et JOBBER D. (2000), « Environmentally responsible purchase behaviour: a test of a consumer model », European Journal of Marketing, Vol. 34, n5/6, pp.723746.

FRANÇOIS-LECOMPTE A. (2006), " La consommation socialement responsable : proposition d'un modèle intégrateur ", Actes du XXII Congrès de l'AFM, Nantes.

GIANNELLONI J.-L. (1998), « Les comportements lies à la protection de l'environnement et leurs déterminants : un état des recherches en marketing ", Recherche et Applications en Marketing, Vol. 13, $\mathrm{n}^{\circ} 2$, pp.49-72.

HOFFMANN G. et SAULQUIN J.Y. (2009), « Quand la RSE revisite la chaîne de valeur », Revue Management \& Avenir, $\mathrm{n}^{\circ} 28$, pp.37-55.

JOANNIS H. (1965), De l'étude de motivation à la creation publicitaire et à la promotion des ventes, Dunod, Paris.

JONES T.M. (1991), « Ethical decision Making by Individuals in Organizations: An IssueContingent Model », The Academy of Management Review, Vol. 16, n², April, pp. 366395.

KILBOURNE W.E. (1995), "Green Advertising: Salvation or Oxymoron? », Journal of Advertising, Vol. 24, $\mathrm{n}^{\circ} 2$, pp. 7-20.

KINNEAR T.C., TAYLOR J.R. et AHMED S.A. (1974), « Ecologically concerned consumers: who are they? ", Journal of Marketing, Vol. 38, April, pp.20-24.

KOVARSKI O. et RENAULT S. (2000), « La consommation écologiquement responsable », Cahier de recherche, Juin, ESC Normandie.

KRÉZIAK D. (1998), " Les motivations de la consommation verte : une approche par les chaînages cognitifs ", Thèse de doctorat, Université Pierre Mendès-France, ESA Grenoble.

KRÉZIAK D. et VALETTE-FLORENCE P. (1997), " Les objectifs poursuivis par les consommateurs écologiquement responsables : une nouvelle approche ", Actes du $13^{\text {ème }}$ Congrès de l'AFM, Toulouse, pp. 135-162.

MILES M. et HUBERMAN A. (2003), Analyses des données qualitatives, De Boeck université, Paris.

PACITTO J.C. et JULIEN P.A. (2004), « Le marketing est-il soluble dans la très petite entreprise ", 7ème Congrès International Francophone en Entrepreneuriat et PME, Montpellier, Octobre, pp. 27-29.

PATUREL R. et RICHOMME-HUET K. (2007), « Le devenir de l'activité artisanale passe-til par l'activité entrepreneuriale ? ", Revue de l'entrepreneuriat, Vol. 6, n¹, pp. 29-52.

PICARD C. (2006), " La représentation identitaire de la TPE artisanale », Revue Internationale PME, Vol. 19, $\mathrm{n}^{\circ} 3-4, \mathrm{pp} .77-110$.

ROBINOT E. et GIANNELLONI J.-L. (2009), « La prise en compte de l'environnement naturel dans les services. Une échelle d'attitude », Recherche et Applications en Marketing, Vol. 24, n², pp.29-51.

ROY A. (1994), "Correlates of mall visit frequency », Journal of Retailing, Vol. 70, Summer, pp.139-162.

SCHWARTZ S.H. et BILSKY W. (1987), « Toward a universal psychological structure of human values ", Journal of Personality and Social Psychology, Vol. 55, n³, pp.550-562. SENNES V., FELONNEAU M.L., RIBEYRE F. et GOMBERT S. (2007), " L'écosphère familiale, un espace clé pour la consommation responsable ", publication en ligne consultée en juin 2010 à l'adresse suivante: http://www.egid.u-bordeaux3.fr/index. php?page=recherche_ades

SPIGGLE S. (1994), "Analysis and Interpretation of Qualitative Data in Consumer 


\section{L'éco-construction quel positionnement pour les artisans?}

Research", Journal of Consumer Research, 21, 491-503

STRAUGHAN R.D. et ROBERTS J.A. (1999), « Environmental segmentation alternatives: a look at green consumer behaviour in the new millennium ", The Journal of Consumer Marketing, Vol. $16, \mathrm{n}^{\circ} 6$, pp.558-575.

SUCHARD H.T. et POLONSKY M.J. (1991), «A theory of environmental buyer behaviour and its validity: the environmental action-behaviour model ", AMA Summer Educators' Proceedings, Vol. 2, pp.187-201.

SUIRE R. (2004), "Des réseaux de l'entrepreneur aux ressorts du créatif : quelles stratégies pour les territoires ? ", Revue Internationale PME, Vol. 17, n², pp.123-144.

TAYLOR D.W. et WALLEY E.E. (2004), « The Green Entrepreneur: Opportunist, Maverick or Visionary? ", International Journal of Entrepreneurship and Small Business, Vol.1, $\mathrm{n}^{\circ} 1 / 2, \mathrm{pp} .56-69$.

THØGERSEN J. et ÖLANDER F. (2003), « Spillover of environment-friendly consumer behaviour », Journal of Environmental Psychology, Vol. 23, n³, pp.225-236.

WACHEUX F. (1996), Méthodes qualitatives et recherche en gestion, Economica, Paris. 
